\title{
ssDNA templated self-assembly of chromophores
}

\section{Supporting information}

Pim G. A. Janssen, Joke Vandenbergh, Joost L. J. van Dongen, E. W. Meijer* and Albertus P. H. J. Schenning*

Contribution from the Laboratory of Macromolecular and Organic Chemistry, Eindhoven University of Technology, P.O. Box 513, 5600 MB Eindhoven, The Netherlands; e.w.meijer@tue.nl; a.p.h.j.schenning@tue.nl

\section{General methods}

${ }^{1} \mathrm{H}$ NMR and ${ }^{13} \mathrm{C}$ NMR were recorded at room temperature on a Varian 300 or Varian Mercury 400. Chemical shifts are given in ppm $(\delta)$ relative to tetramethylsilane. Abbreviations used are $\mathrm{s}=$ singlet, $\mathrm{d}=\mathrm{doublet}$, $\mathrm{dd}=$ double doublet, $\mathrm{t}=$ triplet and $\mathrm{m}=$ multiplet. Infrared (IR) spectra were run on a Perkin Elmer 1600 FT-IR spectrometer. MALDI-TOF MS spectra were measured on a Perspective DE Voyager spectrometer utilising an $\alpha$ cyano-4-hydroxycinnamic acid matrix. Elemental analyses were carried out on a Perkin Elmer 2400. UV-vis and fluorescence spectra were performed on a Perkin Elmer Lambda 40 spectrophotometer and Perkin Elmer LS-50 B. $\mathrm{CD}, \mathrm{UV}$-vis and fluorescence spectra were recorded on a JASCO 815 equiped with a fluorescence detector, JWFMO-427S, and a Peltier temperature controller, PFD-425S. High resolution electrospray ionization mass spectrometry was performed on Q-Tof Ultima Global mass spectrometer (Micromass, Manchester UK) equipped with a Z-spray source. Electrospray ionization was achieved in the negative mode by direct and continuous injection of the $0.5-4 \mathrm{mM}$ solutions in MilliQ water at room temperature with $5 \mu \mathrm{l} / \mathrm{s}$ and by applying $5 \mathrm{kV}$ on the needle. The collision cell was flowed with argon as the collision gas and the cone voltage was set to $10 \mathrm{kV}$.

\section{Sample preparation}


All NT samples were prepared by adding a dTn in $10 \mathrm{mM}$ triethyl ammonium acetate (TEAA) buffer at $\mathrm{pH}=5.2$ to solid NT and subsequent sonification at $70{ }^{\circ} \mathrm{C}$ for at least 5 minutes. In case of OPVT, a concentrated solution in HPLC grade THF was injected into the dTn solution in MilliQ water and the excess of THF was removed by heating the sample to $70^{\circ} \mathrm{C}$. For the titration experiments dTn solution were mixed with the NT-dTn solution. Before annealing these at $4{ }^{\circ} \mathrm{C}$ for at least $24 \mathrm{~h}$, they were heated to above $70{ }^{\circ} \mathrm{C}$ to erase all memory effects. To prevent de-aggregation, the mixtures were quickly transferred into a cuvet and put into the CD-spectrometer at -10 ${ }^{\circ} \mathrm{C}$. When the CD-signal at $269 \mathrm{~nm}$ was equilibrated, two spectra were recorded.

\section{Materials}

Tetraethylene glycol methyl $p$-tosyl ether, $\mathbf{1}^{\mathrm{S} 1}, 2,5$-Bis[(S)-2-methylbutoxy]-1,4-diformylbenzene, 4, diethyl-4cyanobenzyl phosphonate, 5, and 3,4,5-tris(methyltetraethyleneoxy)benzyl chloride, $\mathbf{6}$, were synthesized according to literature proceedings. dT10, dT40 and dA40 were supplied freeze-dried and HPLC purified by MWG Biotech AG. Biobeads SX-3 was obtained from Bio-Rad. All the solvents purchased from Acros Chimica, Aldrich or Fluka were of p.a. quality. Dry DMF and THF were obtained after distillation. Deuterated solvents were obtained from Cambridge Isotope Laboratories. All other chemicals are commercially available and used without further purification.

\section{Synthesis}

NT was synthesized via a Williamson etherification of 6-cyano-2-naphthol, 2, with glycol methyl p-tosyl ether, 1, and a subsequent reaction with dicyandiamide in methoxy-ethanol.

Scheme 1. Synthetic route to NT.
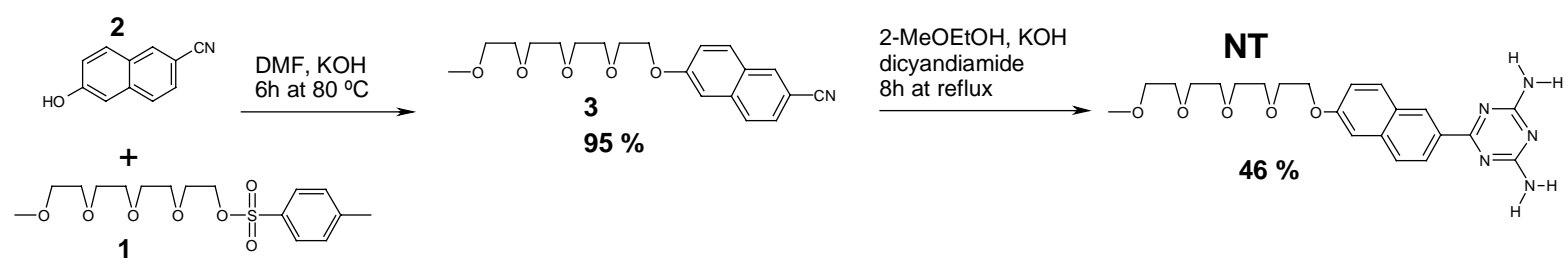

Tetraethylene glycol methyl-6-cyano-2-naphthyl ether 3 
Under argon atmosphere at $0{ }^{\circ} \mathrm{C}$ tetraethylene glycol methyl p-tosyl ether, 1, $(1.75 \mathrm{~g}, 4.83 \mathrm{mmol})$ in $5 \mathrm{ml}$ dry DMF was added dropwise to a solution of 6-cyano-2-naphthol, 2, (817 mg, $4.83 \mathrm{mmol})$ and $\mathrm{KOH}$ (800 mg, $14.3 \mathrm{mmol})$ in dry DMF (20 ml). After 15 minutes the reaction mixture was heated to $80{ }^{\circ} \mathrm{C}$ for $6 \mathrm{hr}$. The reaction mixture was added to $40 \mathrm{ml} 1 \mathrm{~N} \mathrm{HCl}$ and extracted with chloroform $(4 \times 100 \mathrm{ml})$. The collected organic phase was washed neutral with water and dried with $\mathrm{MgSO}_{4}$. Tetraethylene glycol methyl-6-cyano-2-naphthyl ether 3 (1.65 g, $95 \%$ yield) was obtained pure as a white solid after columnar chromatography (active neutral alumina oxide, chloroform: methanol $=$ from $100: 0$ to $98: 2) .{ }^{1} \mathrm{H}$ NMR $\delta\left(400 \mathrm{MHz}, \mathrm{CDCl}_{3}\right) 3.37\left(\mathrm{~s}, 3 \mathrm{H}, \mathrm{OCH}_{3}\right), 3.54(\mathrm{t}, 2 \mathrm{H}$ $\left.\mathrm{OCH}_{2}\right) 3.63-3.71\left(\mathrm{~m}, 8 \mathrm{H}, \mathrm{OCH}_{2}\right), 3.76\left(\mathrm{t}, 2 \mathrm{H}, \mathrm{OCH}_{2}\right), 3.94\left(\mathrm{t}, 2 \mathrm{H}, \mathrm{OCH}_{2}\right), 4.27\left(\mathrm{t}, 2 \mathrm{H}, \mathrm{OCH}_{2}\right), 7.16(\mathrm{~d}, 1 \mathrm{H}, \mathrm{NaH}$, $\mathrm{J}=2.4 \mathrm{~Hz}), 7.27(\mathrm{~d}, 1 \mathrm{H}, \mathrm{NaH}, \mathrm{J}=4.8), 7.55\left(\mathrm{dd}, 1 \mathrm{Na} H, \mathrm{~J}_{1}=2.2, \mathrm{~J}_{2}=8.6\right) 7.76(\mathrm{~d}, 1 \mathrm{H}, \mathrm{Na} H, \mathrm{~J}=3.6 \mathrm{~Hz}) 7.78(\mathrm{~d}$, $1 \mathrm{H}, \mathrm{NaH}, \mathrm{J}=4.4), 8.13(\mathrm{~s}, 1 \mathrm{H}, \mathrm{NaH})$ MALDI-TOF MS $(\mathrm{M}=359.17) \mathrm{M} / \mathrm{z}=[\mathrm{M}]^{+}$; Anal. Calcd for $\mathrm{C}_{20} \mathrm{H}_{25} \mathrm{NO}_{5}: \mathrm{C}_{\text {, }}$ 66.84 H, 7.01. Found: C 66.85, H 7.00\%.

\section{Tetraethylene glycol methyl-6-diaminotriazine-2-naphthyl ether (NT)}

Under argon atmosphere $3(1.03 \mathrm{~g}, 2.87 \mathrm{mmol})$ and dicyandiamide $(250 \mathrm{mg}, 2.95 \mathrm{mmol})$ were dissolved in $10 \mathrm{ml}$ 2-methoxyethanol. After adding $\mathrm{KOH}(400 \mathrm{mg}, 7.18 \mathrm{mmol})$ the reaction mixture was stirred overnight at reflux. The mixture was added to $1 \mathrm{~N} \mathrm{HCl}(20 \mathrm{ml})$ and extracted with with chloroform $(4 \mathrm{x} 50 \mathrm{ml})$. The organic layers were combined, washed with water and dried with $\mathrm{MgSO}_{4}$. Columnar chromatography (active neutral alumina oxide, chloroform: methanol $=$ from $100: 0$ to $90: 10$ ) and precipitation from a concentrated chloroform solution added to pentane yielded NT (586 mg, $46 \%$ yield) as a white powder. ${ }^{1} \mathrm{H} \mathrm{NMR} \delta\left(300 \mathrm{MHz}, \mathrm{CDCl}_{3}\right) 3.29(\mathrm{~s}, 3 \mathrm{H}$, $\left.\mathrm{OCH}_{3}\right), 3.46\left(\mathrm{t}, 2 \mathrm{H} \mathrm{OCH} \mathrm{O}_{2}\right) 3.55-3.64\left(\mathrm{~m}, 8 \mathrm{H}, \mathrm{OCH}_{2}\right), 3.68\left(\mathrm{t}, 2 \mathrm{H}, \mathrm{OCH}_{2}\right), 3.85\left(\mathrm{t}, 2 \mathrm{H}, \mathrm{OCH}_{2}\right), 4.18(\mathrm{t}, 2 \mathrm{H}$, $\left.\mathrm{OCH}_{2}\right), 5.6\left(\mathrm{~s}, 4 \mathrm{H}, \mathrm{NH}_{2}\right) 7.06(\mathrm{~s}, 1 \mathrm{H}, \mathrm{NaH}), 7.11\left(\mathrm{dd}, 1 \mathrm{H}, \mathrm{Na} H, \mathrm{~J}_{1}=2.4\right.$ and $\left.\mathbf{J}_{2}=9.2 \mathrm{~Hz}\right) 7.64(\mathrm{~d}, 1 \mathrm{H}, \mathrm{Na} H, \mathrm{~J}=8.4$

$\mathrm{Hz}), 7.74(\mathrm{~d}, 1 \mathrm{H}, \mathrm{Na} H, \mathrm{~J}=9.3 \mathrm{~Hz}) 8.19(\mathrm{~d}, 1 \mathrm{H}, \mathrm{Na} H, \mathrm{~J}=8.4 \mathrm{~Hz}), 8.64(\mathrm{~s}, 1 \mathrm{H}, \mathrm{NaH}){ }^{13} \mathrm{C}-\mathrm{NMR}\left(300 \mathrm{MHz}, \mathrm{CDCl}_{3}\right)$, $\delta$ 0.7, 58.7, 67.1, 69.3, 70.2, $70.3(3), 70.5,71.6,106.2,119.1,125.1,126.3,128.0,130.4,131.3,136.1,157.7$, 167.2, 171.7; IR (KBr): $v / \mathrm{cm}^{-1}=734,763,806,827,863,913,958,975,1061,1092,1177,1219,1258,1371$, 1386, 1401, 1440, 1481, 1500, 1523, 1967, 2032, 2162, 2877, 3162, 3344, 3468. MALDI-TOF MS $(\mathrm{M}=443.22)$ $\mathrm{M} / \mathrm{z}=443.13[\mathrm{M}]^{+}$; Anal. Calcd for $\mathrm{C}_{22} \mathrm{H}_{29} \mathrm{~N}_{5} \mathrm{O}_{5}: \mathrm{C}, 59.58 \mathrm{H}, 6.59$. Found: C 59.62, H $6.70 \%$. 
Via a Wittig-Horner reaction, diethyl-4-cyanobenzyl phosphonate, $\mathbf{5}^{\mathrm{s} 2}$, was coupled assymmetrically to diformylbenzene $4^{\mathrm{S} 3}$, to obtain nitrile 8. Phosphonate 7 was obtained after an Arbuzov reaction of $\mathbf{6}^{\mathrm{S4}}$ with triethylphosphite. A second Wittig-Horner coupling of $\mathbf{8}$ with phosphonate $\mathbf{7}$ and a subsequent isomerization with iodine yielded nitrile 9. In the final step, 9 reacted with dicyandiamide in 2methoxyethanol into OPVT.

Scheme 2. Synthetic route to OPVT.
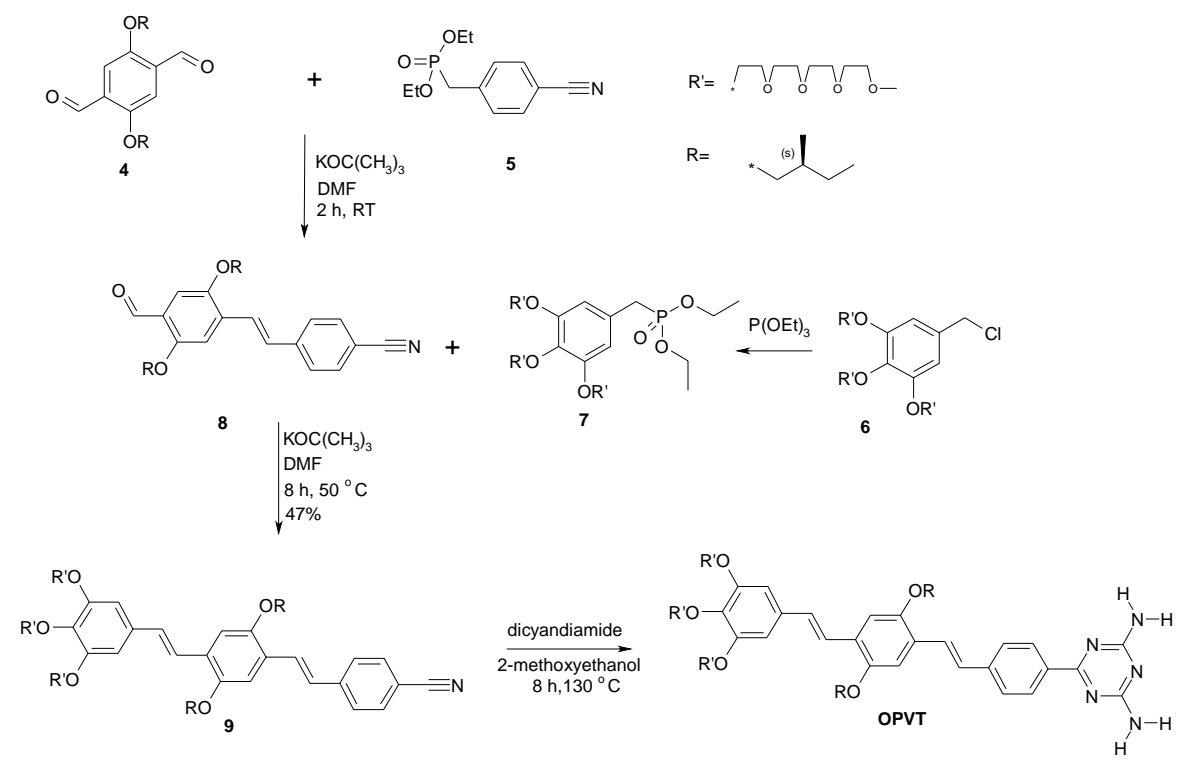

\section{Diethyl(3,4,5-tris(methyltetraethyleneoxy)benzyl)phosphonate 7}

Under an argon atmosphere, a mixture of triethyl phosphite $(1.780 \mathrm{~g}, 10.71 \mathrm{mmol})$ and $6(5.322 \mathrm{~g}, 7.14 \mathrm{mmol})$ was stirred at $160{ }^{\circ} \mathrm{C}$ for $8 \mathrm{~h}$. During this time, ethylchloride was distilled off from the reaction mixture. Subsequently, the mixture was cooled to $70{ }^{\circ} \mathrm{C}$ and the excess of triethylphosphite was distilled off under reduced pressure. Phosphonate 7 was obtained as a light brown clear oil $(5.49 \mathrm{~g}, 91 \%)$ and was used without further purification: ${ }^{1} \mathrm{H}-\mathrm{NMR} \delta\left(300 \mathrm{MHz}, \mathrm{CDCl}_{3}\right) 1.21\left(\mathrm{t}, 6 \mathrm{H}, \mathrm{CH}_{2} \mathrm{CH}_{3}\right), 3.02\left(\mathrm{~d}, 2 \mathrm{H}, \mathrm{ArCH}_{2}\right), 3.36(\mathrm{~s}, 9 \mathrm{H}), 3.50-3.80$ (m, 42H, OCH $\left.\mathrm{OH}_{2}\right), 3.98\left(\mathrm{~m}, 4 \mathrm{H}, \mathrm{OCH}_{2} \mathrm{CH}_{3}\right), 4.10\left(\mathrm{~m}, 6 \mathrm{H}, \mathrm{OCH}_{2}\right), 6.53(\mathrm{~s}, 2 \mathrm{H}, \mathrm{ArH})$.

\section{(E)-4-[4-formyl-2,5-bis[(S)-2-methylbutoxy]styryl]-phenyl nitrile 8}


Under an argon atmosphere, dialdehyde $\mathbf{4}$ and phosphonate 5 were dissolved in $39.7 \mathrm{~mL}$ dry DMF. Potassium tertbutoxide $(0.504 \mathrm{~g}, 4.494 \mathrm{mmol})$ in $27 \mathrm{~mL}$ dry DMF was added slowly to the reaction mixture. After stirring for 2 $\mathrm{h}$, the mixture was poured into $3 \mathrm{~N} \mathrm{HCl}$, extracted with isopropylether and dried over $\mathrm{MgSO}_{4}$. After purification of the crude mixture with column chromatography (silica gel, $\mathrm{CH}_{2} \mathrm{Cl}_{2} /$ heptane $75 / 25$ ), 8 was obtained pure as a yellow powder $(0.61 \mathrm{~g}, 52 \%) .{ }^{1} \mathrm{H}-\mathrm{NMR} \delta\left(300 \mathrm{MHz}, \mathrm{CDCl}_{3}\right): 0.98\left(\mathrm{t}, 6 \mathrm{H}, \mathrm{CH}_{2} \mathrm{CH}_{3}\right), 1.06\left(\mathrm{~d}, 6 \mathrm{HCHCH}_{3}\right), 1.31$ (m, 2H, $\left.\mathrm{CH}_{2} \mathrm{CH}_{3}\right), 1.57\left(\mathrm{~m}, 2 \mathrm{H}, \mathrm{CH}_{2} \mathrm{CH}_{3}\right), 1.99(\mathrm{~m}, 2 \mathrm{H}, \mathrm{CH}), 4.10\left(\mathrm{~m}, 4 \mathrm{H}, \mathrm{OCH}_{2} \mathrm{CH}\right), 7.16(\mathrm{~d}, 1 \mathrm{H}, \mathrm{ArH}), 7.25(\mathrm{~d}$, $1 \mathrm{H}, \mathrm{CH}=\mathrm{CH}, \mathrm{J}=16.5 \mathrm{~Hz}), 7.34(\mathrm{~s}, 1 \mathrm{H}, \mathrm{ArH}), 7.57(\mathrm{~d}, 1 \mathrm{H}, \mathrm{CH}=\mathrm{CH}), 7.59(\mathrm{~s}, 1 \mathrm{H}, \operatorname{Ar} H), 7.62(\mathrm{~s}, 1 \mathrm{H}, \operatorname{Ar} H), 7.62(\mathrm{~d}$, $1 \mathrm{H}, \operatorname{Ar} H, J=6.3 \mathrm{~Hz}), 7.65$ (s, 1H, $\operatorname{Ar} H), 7.67$ (d, 1H, $\operatorname{Ar} H, J=6.3 \mathrm{~Hz}), 10.50$ (s, 1H, CH=O).

\section{(E,E)-4-[-4-(3,4,5-tris\{methyltetraethyleneoxy\})styryl]-2,5-bis[(S)-2-methylbutoxy]styryl]phenyl nitrile 9}

Phosphonate 7 (700 mg, $0.83 \mathrm{mmol}$ ) was dissolved in $90 \mathrm{~mL}$ anhydrous DMF. Potassium tert-butoxide (407 mg, $3.63 \mathrm{mmol}$ ) was added to the solution under an argon atmosphere. After $15 \mathrm{~min}$, a solution of aldehyde 8 (300 $\mathrm{mg}$, $0.74 \mathrm{mmol}$ ) in $15 \mathrm{~mL}$ dry THF was added dropwise to the reaction mixture. The solution was stirred overnight at room temperature and subsequently poured into $250 \mathrm{~mL} \mathrm{HCl}(6 \mathrm{~N})$. The aqueous mixture was extracted three times with chloroform (200 mL). The collected organic layers were washed with $3 \mathrm{~N} \mathrm{HCl}$ and dried over $\mathrm{MgSO}_{4}$, filtered, and evaporated to dryness. The crude mixture was dissolved in toluene with an iodine crystal. This solution was refluxed overnight under argon atmosphere and subsequently concentrated, dissolved in chloroform and washed with a $1 \mathrm{M} \mathrm{Na}_{2} \mathrm{~S}_{2} \mathrm{O}_{4}$ solution. The chloroform layer was dried with $\mathrm{MgSO}_{4}$, filtered, and evaporated to dryness. Nitrile 9 was treated by column chromatography (silica gel, $\mathrm{CH}_{2} \mathrm{Cl}_{2} /$ methanol 97/3) to obtain $266 \mathrm{mg}$ (33\%) of pure 9 as a yellow wax. ${ }^{1} \mathrm{H}-\mathrm{NMR} \delta\left(400 \mathrm{MHz}, \mathrm{CDCl}_{3}\right): 1.00\left(\mathrm{t}, 6 \mathrm{H}, \mathrm{CH}_{2} \mathrm{CH}_{3}\right), 1.12\left(\mathrm{dd}, 6 \mathrm{H}, \mathrm{CHCH}_{3}\right)$, $1.38\left(\mathrm{~m}, 2 \mathrm{H}, \mathrm{CH}_{2}\right), 1.65\left(\mathrm{~m}, 2 \mathrm{H}, \mathrm{CH}_{2}\right), 1.99(\mathrm{~m}, 2 \mathrm{H}, \mathrm{CH}), 3.36\left(\mathrm{~s}, 9 \mathrm{H}, \mathrm{OCH}_{3}\right), 3.50-4.00\left(\mathrm{~m}, 42 \mathrm{H}, \mathrm{OCH}_{2} \mathrm{CH}_{2}\right)$, $3.80\left(\mathrm{t}, 2 \mathrm{H}, \mathrm{OCH}_{2} \mathrm{CH}\right), 4.19\left(\mathrm{t}, 2 \mathrm{H}, \mathrm{OCH}_{2} \mathrm{CH}\right), 4.20\left(\mathrm{t}, 6 \mathrm{H}, \mathrm{ArOCH}_{2}\right), 6.76(\mathrm{~s}, 2 \mathrm{H}, \mathrm{ArH}), 7.04(\mathrm{~d}, 1 \mathrm{H}, \mathrm{CH}=\mathrm{CH})$, $7.08(\mathrm{~s}, 2 \mathrm{H}, \mathrm{ArH}), 7.13(\mathrm{~d}, 1 \mathrm{H}, \mathrm{CH}=\mathrm{CH}), 7.32(\mathrm{~d}, 1 \mathrm{H}, \mathrm{CH}=\mathrm{CH}), 7.57(\mathrm{~d}, 2 \mathrm{H}, \mathrm{ArH}), 7.58(\mathrm{~d}, 1 \mathrm{H}, \mathrm{CH}=\mathrm{CH}), 7.63$ $(\mathrm{d}, 2 \mathrm{H}, \operatorname{Ar} H)$.

\section{2,4-diamino-6-[(E,E)-4-[-4-(3,4,5-tris\{methyltetraethyleneoxy\})styryl]-2,5-bis[(S)-2-methylbutoxy]- styryl]phenyl]-s-triazine OPVT}


$260 \mathrm{mg}(0.242 \mathrm{mmol})$ of $\mathbf{1 2}$ and $26 \mathrm{mg}(0.266 \mathrm{mmol})$ of dicyandiamide were dissolved in $5 \mathrm{~mL} \mathrm{2-}$ methoxyethanol. After adding $35 \mathrm{mg}(0.62 \mathrm{mmol})$ of $\mathrm{KOH}$, the solution was stirred for $8 \mathrm{~h}$ and subsequently poured into $25 \mathrm{~mL} \mathrm{HCl}(6 \mathrm{~N})$. The aqueous mixture was extracted three times with chloroform $(50 \mathrm{~mL})$. The collected organic layers were washed with $3 \mathrm{~N} \mathrm{HCl}$ and dried over $\mathrm{MgSO}_{4}$, filtered, and evaporated to dryness. OPVT (70 mg, 24\%) was obtained as a pure waxy solid after column chromatography (silica gel, $\mathrm{CH}_{2} \mathrm{Cl}_{2} / \mathrm{EtOH}$ 95:5 and BioBeads SX-3). was obtained. 1H-NMR $\delta\left(400 \mathrm{MHz}, \mathrm{CDCl}_{3}\right): 1.00\left(\mathrm{t}, 6 \mathrm{H}, \mathrm{CH}_{2} \mathrm{CH}_{3}\right), 1.12(\mathrm{dd}, 6 \mathrm{H}$, $\left.\mathrm{CHCH}_{3}\right), 1.38\left(\mathrm{~m}, 2 \mathrm{H}, \mathrm{CH}_{2}\right), 1.65\left(\mathrm{~m}, 2 \mathrm{H}, \mathrm{CH}_{2}\right), 1.99(\mathrm{~m}, 2 \mathrm{H}, \mathrm{CH}), 3.36\left(\mathrm{~s}, 9 \mathrm{H}, \mathrm{OCH}_{3}\right), 3.50-4.00(\mathrm{~m}, 42 \mathrm{H}$, $\left.\mathrm{OCH}_{2} \mathrm{CH}_{2}\right), 3.80\left(\mathrm{t}, 2 \mathrm{H}, \mathrm{OCH}_{2} \mathrm{CH}\right), 4.19\left(\mathrm{t}, 2 \mathrm{H}, \mathrm{OCH}_{2} \mathrm{CH}\right), 4.20\left(\mathrm{t}, 6 \mathrm{H}, \mathrm{ArOCH}_{2}\right), 5.15\left(\mathrm{~s}, 4 \mathrm{H}, \mathrm{NH} H_{2}\right), 6.76(\mathrm{~s}, 2 \mathrm{H}$, $\operatorname{ArH}), 7.04(\mathrm{~d}, 1 \mathrm{H}, \mathrm{CH}=\mathrm{CH}), 7.09(\mathrm{~s}, 1 \mathrm{H}, \mathrm{ArH}), 7.13(\mathrm{~s}, 1 \mathrm{H}, \mathrm{ArH}), 7.2(\mathrm{~d}, 1 \mathrm{H}, \mathrm{CH}=\mathrm{CH}), 7.34(\mathrm{~d}, 1 \mathrm{H}, \mathrm{CH}=\mathrm{CH})$, $7.58(\mathrm{~d}, 1 \mathrm{H}, \mathrm{CH}=\mathrm{CH}), 7.60(\mathrm{~d}, 2 \mathrm{H}, \mathrm{ArH}), 8.32(\mathrm{~d}, 2 \mathrm{H}, \mathrm{ArH}) . \mathrm{IR}(\mathrm{KBr}): v / \mathrm{cm}^{-1}=695,815,851,903,959,1040$, 1103, 1200, 1246, 1299, 1331, 1349, 1394, 1433, 1504, 1535, 1579, 1605, 1977, 2160, 2874, 3061, $3217,3343$. MALDI-TOF MS $(\mathrm{M}=1181.67) \mathrm{M} / \mathrm{z}=1181.60[\mathrm{M}]^{+}$; Anal. Calcd for $\mathrm{C}_{62} \mathrm{H}_{95} \mathrm{~N}_{5} \mathrm{O}_{17}$ : $\mathrm{C}, 62.98 \mathrm{H}, 8.10$. Found: $\mathrm{C}$ 62.90, H $8.08 \%$.
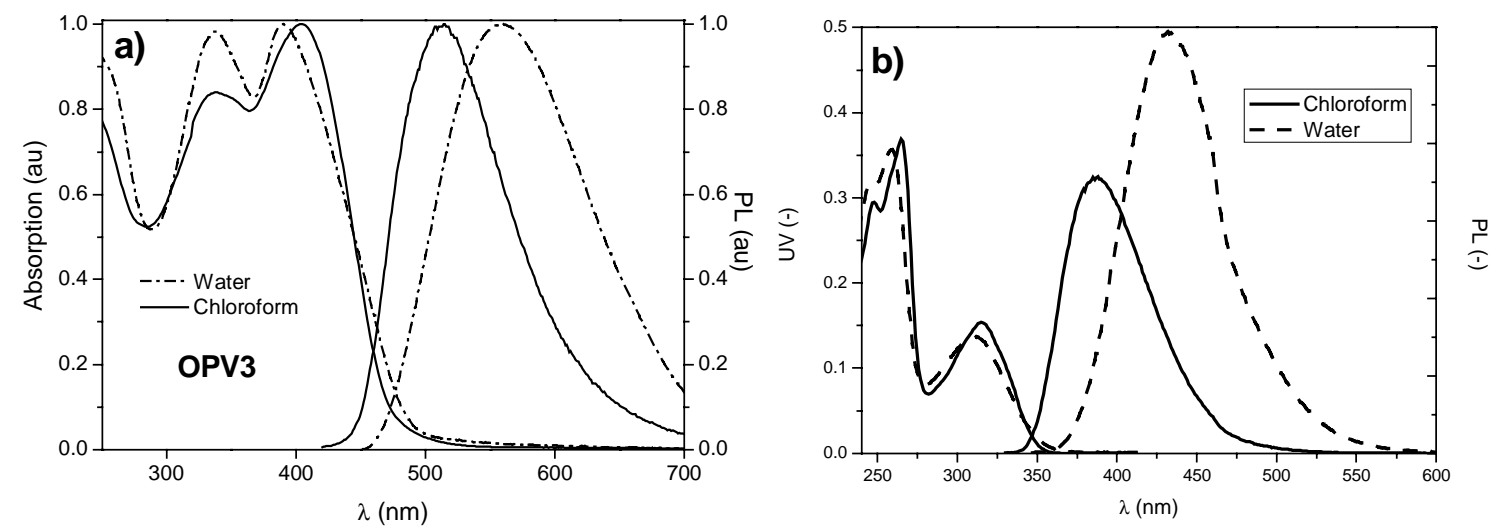

Figure S1. (a) Normalized UV-vis and fluorescence spectra of OPVT in water and chloroform. (b) UV-vis and fluorescence spectra of NT in water and chloroform, $[\mathbf{N T}]=0.2 \mathrm{mM}$. 

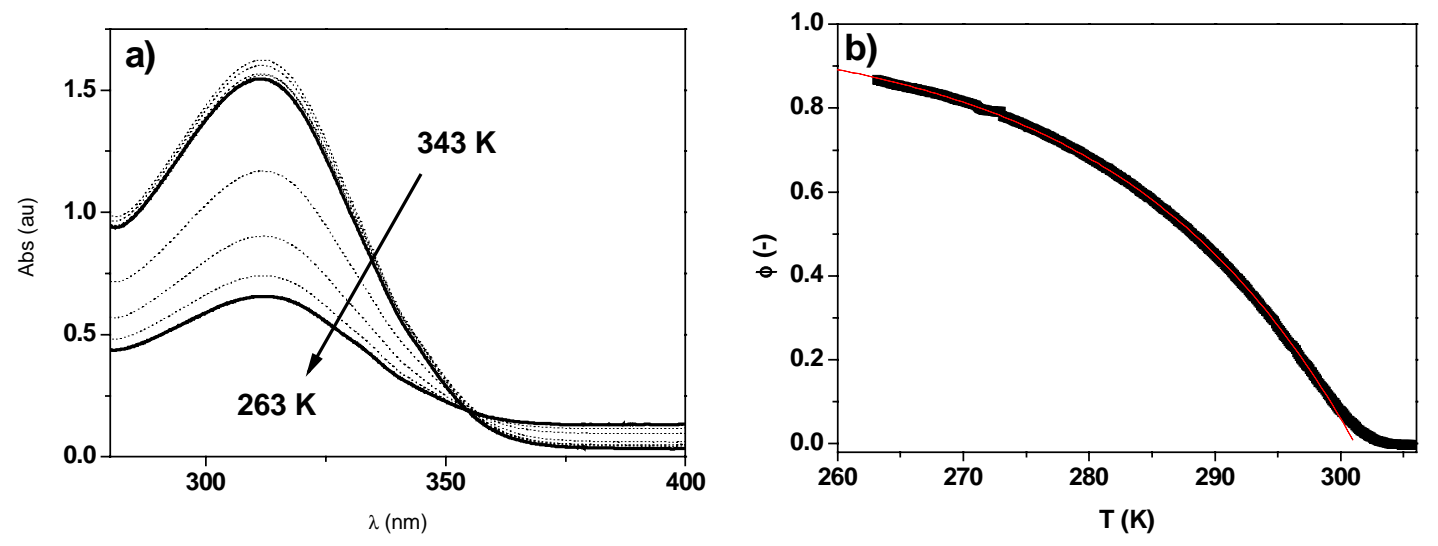

Figure S2. (a) Temperature dependent UV-vis spectra of a $1.25 \mathrm{mM}$ NT mixture in water from 343 to $263 \mathrm{~K}$. (b) Temperature dependent UV-vis intensity at $311 \mathrm{~nm}$ fit to the cooperative supramolecular polymerization model (red line). ${ }^{\mathrm{S} 5}$
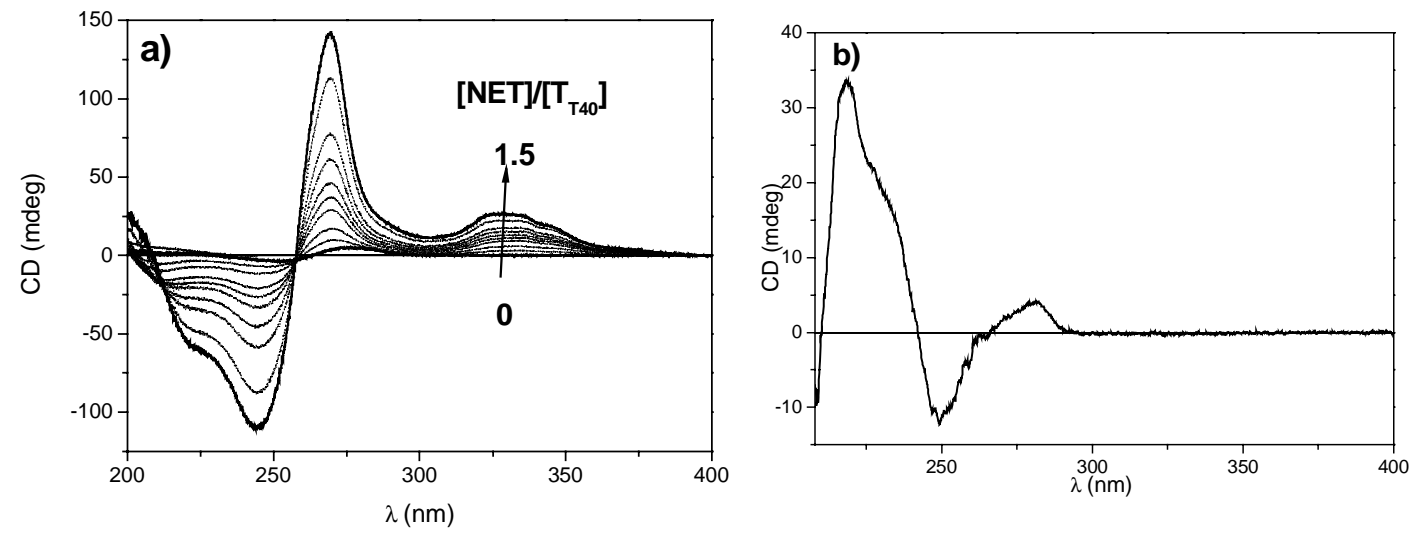

Figure S3. (a) CD-spectra of the titration of NT to dT40, with [dT40] $=8.5 \mu \mathrm{M}$ and $[\mathbf{N T}]$ increasing from 0 to $0.5 \mathrm{mM}$. (b) CD-spectrum of $\mathbf{d A 4 0}(12.5 \mu \mathrm{M})$ and NT $(0.5 \mathrm{mM})$ in water (10 mM TEAA, 10 $\mathrm{mM} \mathrm{MgCl} 2$ ) at $263 \mathrm{~K}$.
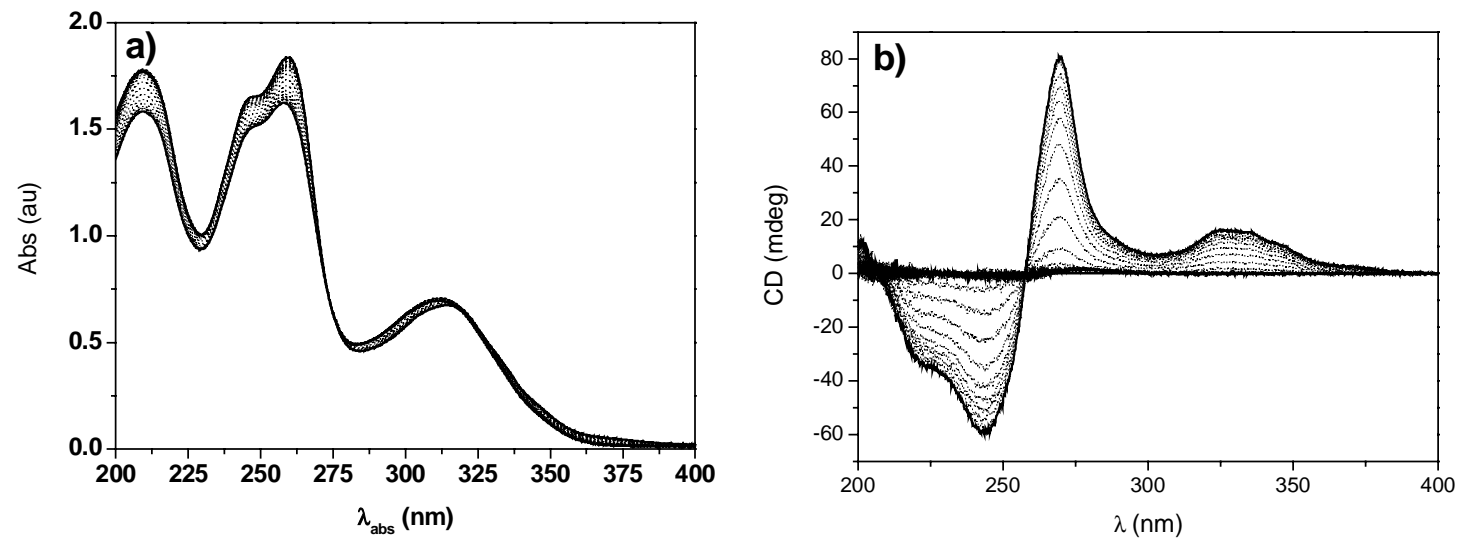
Figure S4. Temperature dependent UV-vis (a) and CD (b) spectra of a dT10 - NT mixture in water. $[\mathbf{N T}]=0.2 \mathrm{mM}$

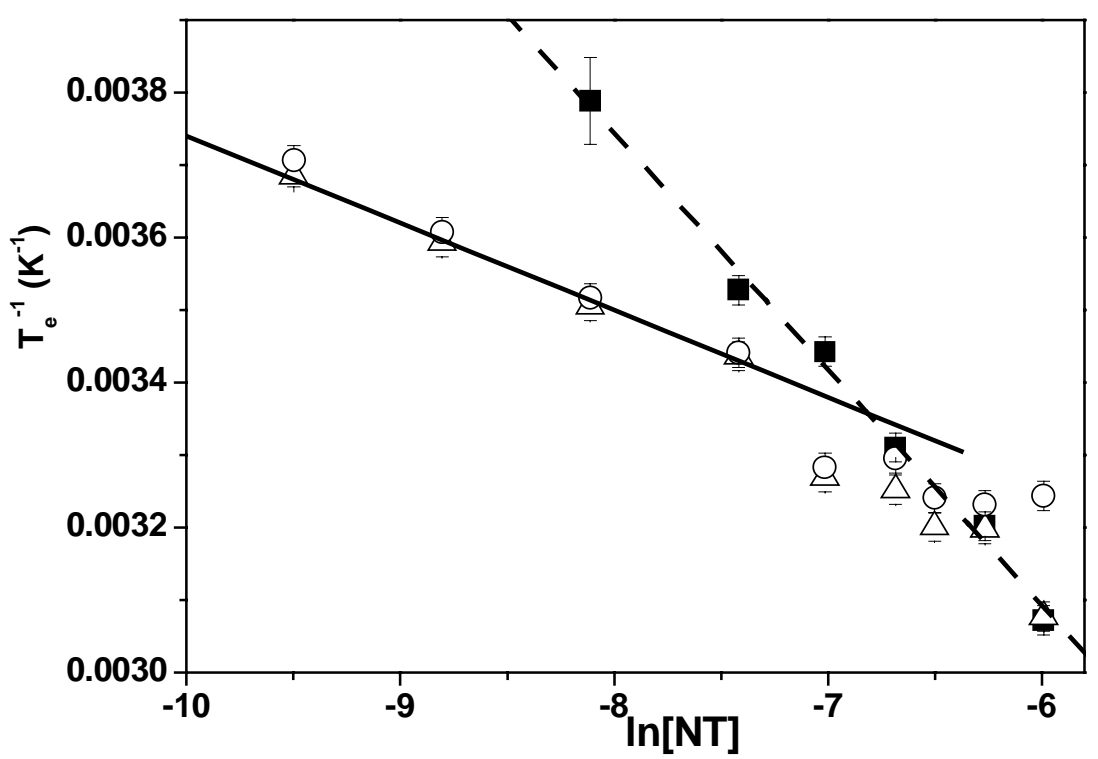

Figure S5. Plot of $\mathrm{T}_{\mathrm{e}}^{-1}$ against $\ln [\mathbf{N T}]$ of NT-dT40 (absorption $(\Delta$, solid line), CD (o)) mixtures (40:1) and of NT alone (absorption ( $\mathbf{\square}$, dashed line)).
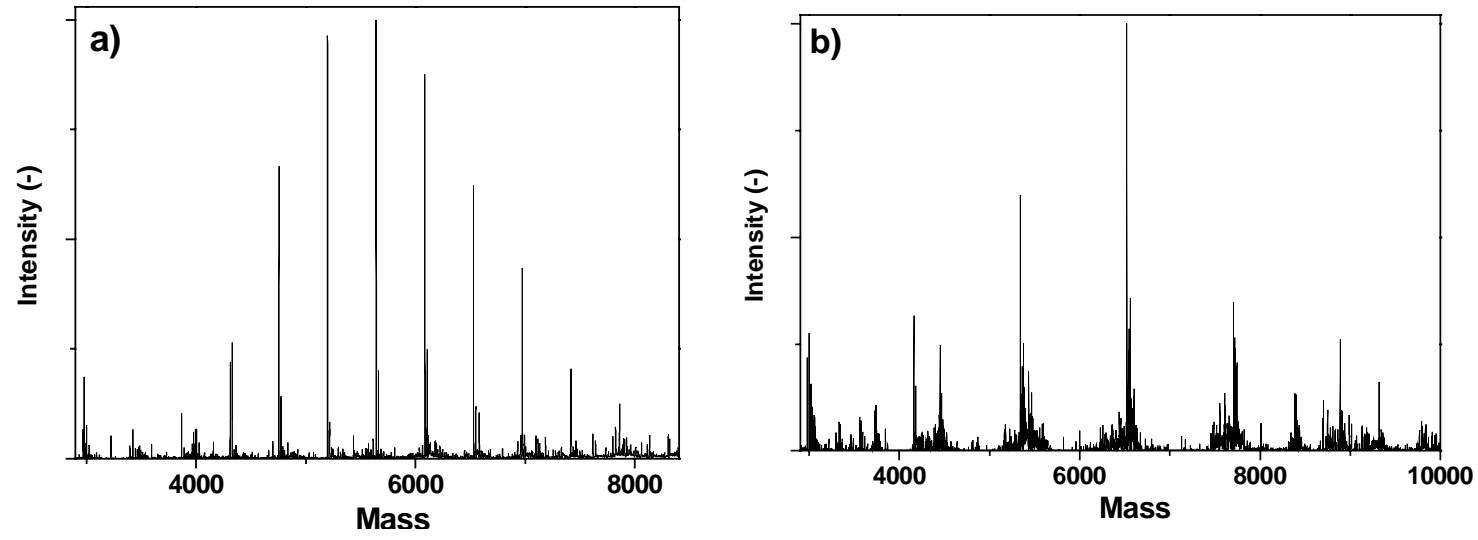

Figure S6. Deconvoluted ESI-MS spectra of the dT10-NT (a) and dT10-OPVT (b) complexes. 
Table S1. Calculated average masses (Isopro 3.0) of dT10-nNT, and dT10-nOPVT complexes and average masses found after deconvolution, with $n=1-11=$ number of NT or OPVT bound to dT10 and with $*=$ not detected.

\begin{tabular}{|c|c|c|c|c|}
\hline $\mathrm{n}$ & $\mathrm{M}_{(\mathbf{d T 1 0}-\mathrm{nNT})}$ & Found & $\mathrm{M}_{(\mathbf{d T 1 0}-\mathrm{nOPVT})}$ & Found \\
\hline 0 & 2979.99 & 2980 & 2979.99 & 2980 \\
\hline 1 & 3423.50 & 3424 & 4162.46 & 4162 \\
\hline 2 & 3867.00 & 3867 & 5344.95 & 5345 \\
\hline 3 & 4310.50 & 4311 & 6527.39 & 6527 \\
\hline 4 & 4754.02 & 4754 & 7709.85 & 7709 \\
\hline 5 & 5197.52 & 5198 & 8892.32 & 8892 \\
\hline 6 & 5641.02 & 5641 & 10074.78 & $*$ \\
\hline 7 & 6084.53 & 6084 & 11257.25 & $*$ \\
\hline 8 & 6528.03 & 6528 & 12439.71 & $*$ \\
\hline 9 & 6971.54 & 6971 & 13622.17 & $*$ \\
\hline 10 & 7415.03 & 7415 & 14804.64 & $*$ \\
\hline 11 & 7858.54 & 7859 & 15987.10 & $*$ \\
\hline
\end{tabular}

S1 Schultz, R. A.; White, B. D.; Dishong, D. M.; Arnold, K. A.; Gokel, G. W. J. Am. Chem. Soc. 1985, 107, 6659 - 6668 .

S2 Hulvat, J. F.; Sofos, M.; Tajima, K.; Stupp, S. I. J. Am. Chem. Soc. 2005, 127, 366-372.

S3 Jonkheijm, P.; Fransen, M.; Schenning, A. P. H. J.; Meijer, E. W. J. Chem. Soc., Perkin Trans. 2 2001, 8, 1280-1286.

S4 Middel, O.; Verboom, W.; Reinhoudt, D. N. Eur. J. Org. Chem. 2002, 2587 - 2597.

S5 (a) Jonkheijm, P.; van der Schoot, P.; Schenning, A. P. H. J.; Meijer, E. W. Science 2006, 313, 8083. (b) Ciferri, A. Supramolecular Polymers, Second Edition, CRC Press LLC: Boca Raton, 2005, pp 77-106. 